\title{
Malvaceae in the flora of Egypt 1. Systematic revision of the indigenous taxa
}

\author{
M. Nabil El-Hadidi \\ Hasnaa A. Hosni \\ Azza M. H. El-Hadidy \\ and
}

Sheriffa Araffa

The Herbarium, Faculty of Science

Cairo University, Giza 12613, Egypt.

El-Hadidi M. N., Hosni H. A., El-Hadidy A. M H. \& Araffa S. Malvaceae in the flora of Egypt. 1. Systematic revision of the indigenous taxa. Taeckholmia 19(2): 127-146.

The idiginous taxa of Malvaceae represented in the flora of Egypt were systematically revised. This revealed the presence of 26 species belonging to 11 genera and four tribes; among these, Malvella sherardiana ia a new record to the flora of this country.

For each species, valid name, synonymes (if any), type, distribution (local and global) and selected specimens are given. A key for the genera of the family, and keys for the species belonging to the genera with more than one species are provided

Key words: Flora of Egypt, Malvaceae, idigenus taxa; Malvella sherardiana.

\section{Introduction}

Malvaceae is a natural family, easily distinguished by its monadelphous stamens, monothecal anthers, simple entire mostly palmate leaves, stellate hairy indumentum and large echinate pollen grains. The presence of an involucre (epicalyx), is diagnostic where present.

Malvaceae is closely related to Tiliaceae, Bombacaceae and Sterculiaceae but differs in the possession of one - celled anthers and monadelphous stamens. It is believed that Malvaceae is regarded as a complex climax derived from the ancestral woody Tiliales and largely reduced to herbs with fibrous stems and is considered as the most advanced family in order Malvales, together with Bombacaceae (Hutchinson, 1967).

Malvaceae is represented by 119 genera and about 2000 species of herbs, shrubs rarely trees, occur overmost of the world except the very cold regions, well represented in the tropical and subtropical regions of the world, particularly abundant in Tropical South America. Some genera show a small area of distribution e.g. the monotype Malvella is Mediterranean. On the other hand, many genera occur throughout the world or throughout the tropics e.g. Hibiscus (300 species). Certain species are of economic value, the most important are Gossypium species, which are the source of cotton fibers and cotton seed oil. Several species are weeds and constitute the natural vegetation of waste places, roadsides while numerous species are naturalised or widely cultivated as oranometals.

Received 21 April 1999. Revision accepted 13 November 1999. 
Malvaceae was the subject of several treatments, the most important are those of Bentham \& Hooker (1862), Schumann (1895) and Hutchinson (1967). The recent treatment carried out by Bremer et al. (1998) based on molecular sequence data particularly from choloroplast DNA rbc L. gene and morphological data; included the tropical and woody groups previously classified in Bombacaceae, Sterculiaceae and Tiliaceae in family Malvaceae (s.1.).

Täckholm (1956) recognized 8 genera comprising 31 species which were increased (Täckholm, 1974) to 10 genera and 32 species. Recently, El Hadidi \& Fayed (1994/95) enumerated 10 genera and 32 species of Malvaceae, a similar number was reported by Boulos (1995).

\section{Systematic Treatment}

\section{Synopsis to the Egyptian taxa of Malvaceae}

The taxa are arranged according to the system proposed by Hutchinson (1967).

A. Tribe : Hibisceae Endl.

1. Hibiscus L.

1. H. trionum L.

2. H. micranthus L.f.

3. H. vitifolius L.

4. H. sabdariffa L.

2. Gossypium L.

5. G. arboreum L.

B. Tribe : Abutileae Hutch.

a. Subtribe : Abutilinae

3. Abutilon Mill.

6. A. theophrasti Medik

7. A. bidentatum Hochst ex A.Rich.

8. A. fruticosum Guill. \& Perr.

9. A. pannosum (G. Forst.) Schledt.

10. A. figarianum Webb.

b. Subtribe : Sidinae (K. Schum.) Hutch.

4. Sida L.

11. S. alba L.

12. S. acuta Burm. fil.

5. Malvella Jaub. \& Spach

* 13. M. sherardiana (L.) Jaub. \& Spach

6. Malvastrum A.Gray

14. M. coromandelianum (L.) Garke

* New record to the flora of Egypt. 


\section{Tribe : Malveae A. Gray \\ Subtribe : Malvinae \\ 7. Malva L. \\ 15. M. aegyptia L. \\ 16. M. parviflora L. \\ 17. M. nicaeensis All. \\ 18. M. neglecta Wallr. \\ 19. M. sylvestris L.}

8. Althaea L.

20. A. ludwigii L.

9. Alcea L.

21. A. striata (DC.) Alef.

22. A. acaulis (Cav.) Alef.

23. A. rosea $\mathrm{L}$.

10. Lavatera L.

24. L. cretica $\mathrm{L}$.

D. Tribe : Ureneae Benth. \& Hook.

11. Pavonia Cav.

25. P. kotschyi Hochst ex Webb.

26. P. triloba Guill. \& Perr.

\section{Key to the genera of Malvaceae}

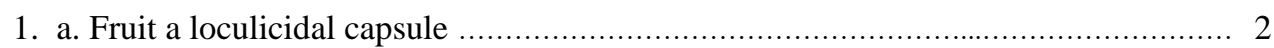

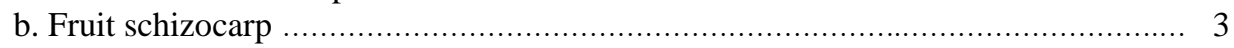

2. a. Bracteoles 3, broadly ovate - cordate, apically toothed, styles united ... 2. Gossypium
b. Bracteoles numerous, filiform linear, entire, styles free
1. Hibiscus

3. a. Bracteoles absent, mericarps dehiscent into 2 valves each ............................... 4

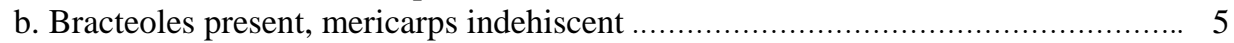

4. a. Mericarp glabrous, one - seeded ................................................ 4. Sida

b. Mericarp hairy, 2 - 3 seeded ................................................ 3. Abutilon

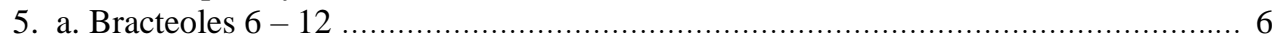

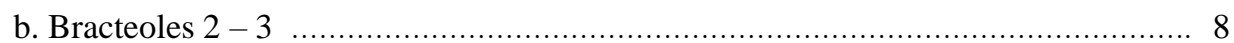

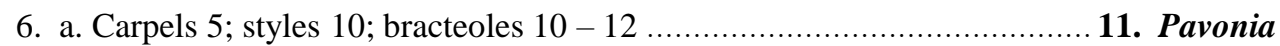

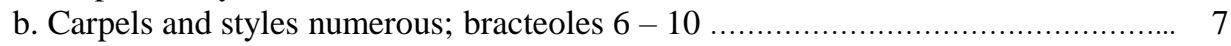

7. a. Bracteoles $8-10$; mericarps 10 , arranged around a short conical axis; flowers $0.6-1 \mathrm{~cm}$ diam; petals up to $1.6 \mathrm{~cm}$ long

8. Althaea

b. Bracteoles 6-7; mericarps more than 20, arranged around a dilated brood dentate axis; flowers $2-6 \mathrm{~cm}$ diam; petals $2-4 \mathrm{~cm}$ long .................... 9. Alcea

8. a. Bracteoles connate at base ................................................. 10. Lavatera

b. Bracteoles free ............................................................................... 9

9. a. Mericarps inflated; petals entire …........................................ 5. Malvella

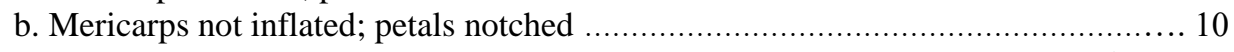

10.a. Pedicel short up to $5 \mathrm{~mm}$ long; stigma capitate ............................ . Malvastrum

b. Pedicel long, $10-15 \mathrm{~mm}$; stigma filiform ............. ... 7. Malva 


\section{Systematic revision}

The present revision is based on collections kept in Cairo University Herbarium (CAI), and the Herbarium of Agricultural Research Centre (CAIM); as well as intensive field studies. Phytogeographical territories for the selected specimens are those proposed by El Hadidi (1980), Fig. (1).

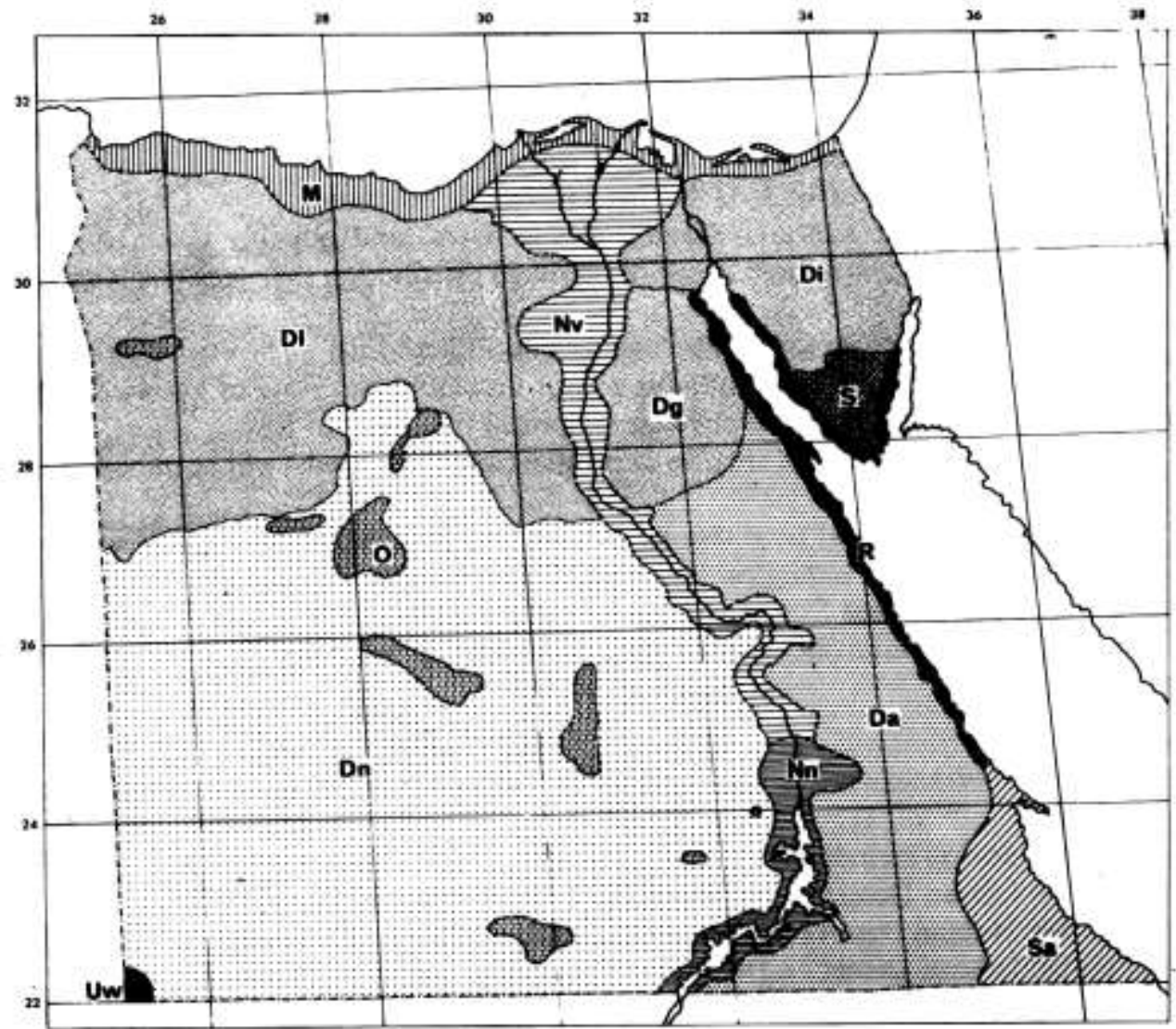

Fig. (1). Phytogeographical subdivisions of Egypt (after El Hadidi, 1980). (M) Mediterranean coastal belt, (D1) Libyan Desert, (Dn) Nubian Desert, (Di) Isthmic Desert, (Dg) Galala Desert, (Da) Arabian Desert, (Nv) Nile Valley sector of the Nile-land, (Nn) Nile nubian sector of the Nile land, (O) Oases of (Dl) \& (Dn), (S) Southern mountainous Sinai, (R) Red Sea coastal plains, (Sa) Gebel Elba district; (Uw) Gebel Uweinat area. 
1. Hibiscus L., Sp. Pl. ed. 1 : 693 (1753), nom. conserv.

Trionum L. Syst. ed. 1 (1735)

Solandra Murr. in comm. Götting. 1783-84 : 21, tab. 1 (1785).

Trionaea Medik., Malv. : 45 (1787).

Abelmoschus Medik., Malv. : 45 (1787).

A large genus of about 300 species widely distributed in tropical and subtropical regions of the world with several cultivated taxa.

\section{Key to the species}

1. a. Leaves undivided, ovate; petals up to $8 \mathrm{~mm}$ long; fruit ovoid, not included in calyx

2. H. micranthus

b. At least upper leaves $3-5$ palmately labed; petals $9-3 \mathrm{~mm}$ long; fruit included in persistent calyx

2. a. Epicalyx segments lanceolate; flower pedicel up to $2 \mathrm{~cm}$ long; seeds hispid

4. H. sabdariffa

b. Epicalyx segments filiform; flower pedicel $2-6 \mathrm{~cm}$ long; seeds tuberculate.......... 3

3. a. Fruit spheroidal, $1 \mathrm{~cm}$ diam, beaked and winged 3. H. vitifolius

b. Fruit oblong - ovoid, $1.5 \times 1 \mathrm{~cm}$, obtuse, wingless 1. H. trionum

1.1. Hibiscus trionum L., Sp. Pl. ed. 1 : 694 (1753); Forssk., Fl. Aegypt. Arab. CXVI no. 424 (1775); Boiss., Fl. Orient. 1 : 840 (1867); Asch. \& Schweinf., Ill. Fl. Égypte : 52 no. 213 (1887); Sickenb., Mém. Inst. Égypt. 4 (2) : 191 (1901); Muschl., Man. Fl. Egypt 1 : 634 (1912); Ramis, Best. - Tabell Fl. Aegypt. : 134 (1929); Täckh., Stud. Fl. Egypt ed. 1 : 232 (1956); et ed. 2 : 356 (1974).

Type: Described from Italy and Africa, Specimen no. 875/39 (LINN, Photo).

Hibiscus ternatus Cav., Diss. : 172 (1787).

\section{Distribution:}

Common weed in fields and along canal banks in the Mediterranean coastal land and the Nile land of Egypt. Known from the Mediterranean, Middle East, Trop. Africa, Pakistan to Australia and America.

\section{Selected specimens:}

(M) Alexandria, 14.8.1929; Hassib. s.n. (CAI)-(Nv) Fayium, 8.12.1991; S.Araffa s.n. (CAI)-(Nn) kom Ombo, 19.4.1967; Abdel Salam et al. s.n. (CAI).

1.2. Hibiscus micranthus L.f. Suppl. Pl. : 308 (1782); Täckh., Stud. Fl. Egypt ed. 1 : 231 (1956); et ed. 2 : 356 (1974).

Type: Specimen no. 872/2 (LINN). 


\section{Distribution:}

Common in rocky and sandy soils of Gebel Elba district, rare along Red Sea Coast and mountainous Sinai. Known from the Sudan, Arabian Peninsula to India and Srilanka.

\section{Selected specimens:}

(S) Baraq El Samrah, 4.5.1972; Kaiser 489 (CAIM)-(R) Gebel Hamatā, 7.2.1961; V.Täckholm et al. s.n. (CAI)- (Sa) Wadi Mawaw, 28.1.1962; V.Täckholm et al. s.n. (CAI).

1.3. Hibiscus vitifolius L., Sp. Pl. ed. 1:696 (1753); Täckh., Stud. Fl. Egypt ed. 1:282 (1956); et ed. 2:356 (1974); Hepper \& Friis, Pl. Forssk. Fl. Aegypt. Arab.:197 (1994).

Type: Herb. Hermann, Vol. IV, Fol. 39, Linn. No. 265 (BM; lectotype selected by Brenan \& Exell : 70, 1958).

Hibiscus tripartitus Forssk., Fl. Aegypt. - Arab. : 126, CXVII no. 422, IV no. 96 (1775); Hepper \& Friis, Pl. Forssk. Fl. Aegypt. - Arab. : 197 (1994).

\section{Distribution:}

Rare in rocky and sandy soils of Gebel Elba district. Known from Trop. Africa, Arabian Peninsula to India and Australia.

\section{Selected specimens:}

(Sa) Wadi Yahameib, 22.1.1962; V.Täckholm et al. s.n. (CAI) - Gebel Elba, 28.2.1938; Shabetai z 5793 (CAIM).

1.4. Hibiscus sabdariffa L., Sp. Pl. ed. 1 : 695 (1753); Muschl., Man. Fl. Egypt 1 : 635 (1912); Täckh., Stud. Fl. Egypt ed. 1 : 232 (1956); et. ed 2 : 268 (1974).

Type: Specimen no. 875/27 (LINN).

Hibiscus gosseypifolius Mill., Gard. Dict. ed. 8 : 10 (1768).

H. digitatus Cav., Diss. 3 : 151, tab. 10, fig. 2 (1787)

\section{Distribution:}

Cultivated in Upper Egypt and the Oases, else escaped and naturalized. Native in India, widespread by introduction in the tropics as a medicinal plant and the source of a refreshing drink. 


\section{Selected specimens:}

(Nv) North garden, El Saff, 27.10.1961; V.Täckholm s.n. (CAI)-(Nn) Plant Island, Asuan, 21.10.1965; El Mahdi s.n. (CAI).

2. Gossypium L. Sp. Pl. ed. 1 : 693 (1753).

Xylon Mill., Gard. Dict. Abr. Ed. 4 : 3 (1754)

Sturtia R.Br. in Sturt, Exped. Gen. Austral. 2 : app. 68 (1849).

A genus of about 20 species in warm regions of both hemispheres; several species are with large number of horticultural cultivars and hybrids

2.1. Gossypium arboreum L., Sp. Pl. ed. 1 : 693 (1753); Forssk., Fl. Aegypt. - Arab. : LXX no. 341; CXVI no. 417 (1775); Delile, Descr. Egypte, Hist. Nat. 2 : 69 (1813); Sickenb., Mém. Inst. Égypt. 4 (2) : 195 (1901); Muschl., Man. Fl. Egypt 1: 637 (1912); Ramis, Best. - Tabell. Fl. Aegypt. : 133 (1929); Täckh., Stud. Fl. Egypt ed. 2: 356 (1974); Hepper \& Friis, Pl. Forssk. Aegypt. - Arab. : 229 (1994).

Type: Specimen no. 874/3 (LINN).

\section{Distribution:}

Sand dunes and loamy soil in Siwa and Bahariya Oases, naturalized. Known from Angola, the Sudan, Arabia, Syria, Iran, Indo-China, Burma, Malaysia, Indonesia, China, Korea, Japan, Bangaladish and India. Cultivated in most tropical countries.

\section{Selected specimens:}

(O) Siwa Oasis, Ain Shohba, 18.4.1986; El Hadidi s.n. 26,4 (CAI) - Bahariya Oasis, 15.9.1941; M.Imam s.n. (CAI).

3. Abutilon Mill., Gard. Dict. Abr. Ed. 4 (1754).

A genus of about 100 species, mostly in tropical and subtropical regions of both hemispheres. Several species are cultivated or of economic importance.

Note: Abutilon longicuspe Hochst. ex A. Rich. is a Tropical African species which is expected to occur in the southern parts of the Eastern Desert. Täckholm (1974:354) reported this species from Gebel Elba district without certainity. No specimens were seen from this area.

\section{Key to the species}

1. a. Mericarps rounded at apex; flower pedicel not more than $1 \mathrm{~cm}$ long ................... 2

b. Mericarps acute - acuminate or awned at apex; flower pedicel $2-6 \mathrm{~cm}$ long ......... 3

2. a. Petals $7-9 \mathrm{~mm}$ long, yellow without purple base 5. A. figarianum 
b. Petals $10-25 \mathrm{~mm}$ long, yellow with purple base

4. A. pannosum

3. a. Mericarp 2 - awned; leaves subentire to slightly dentate

b. Mericarp with acute - acuminate apex; leaves sharply dentate 4

4. a. Mericarps 10, each with acute apex; fruit $1.2 \mathrm{~cm}$ diam

b. Mericarps 20, each with acuminate apex; fruit $0.7 \mathrm{~cm}$ diam

2. A. bidentatum

3.1. Abutilon theophrasti Medik., Malven. : 28 (1787); Täckh., Stud. Fl. Egypt ed. 2 : 354 (1974).

Type: Described from India, Hb. Cliff. (BM).

Sida abutilon L., Sp. Pl. ed. 1 : 685 (1753)

Abutilon avicennae Gaertn., Fruct. Sem. Pl. 2 : 251, tab. 135, fig.1 (1791); Boiss., Fl. Orient. 1 : 836 (1867); Asch. \& Schweinf., Ill. Fl. Égypte : 52 no. 210 (1887); Sickenb., Mém. Inst. Égypt. 4 (2) : 191 (1901); Muschl. Man. Fl. Egypt 1 : 632 (1912); Ramis, Best. - Tabell. Fl. Aegypt. : 134 (1929); Täckh., Stud. Fl. Egypt ed. 1: 229 (1956).

\section{Distribution:}

Naturalized in the moist places and the farmland of the Nile Delta. Known from Mediterranean, SE Europe, eastwards to Iran and Afghanistan.

\section{Selected specimens:}

(M) Ezbet El Awam, Damietta, 13.6.1922; Dsiwfswy 1467 (CAIM) - (Nv) Menoufia province, Ganzour, 15.9.1967; V.Täckholm s.n. (CAI) - Fayium, Ibshwai, 3.6.1983; Abdel Ghani 6490 (CAI).

3.2. Abutilon bidentatum Hochst. ex A. Rich., Tent. Fl. Abyss. 1 : 68 (1847); Asch. \& Schweinf., Ill. Fl. Égypte : 52 no. 209 (1887); Muschl., Man. Fl. Egypt 1 : 631 (1912); Ramis Best. - Tabell. Fl. Aegypt. : 134 (1929); Täckh., Stud. Fl. Egypt ed. 1:230 (1956); et ed. 2 : 354 (1974); Hepper \& Friis, Pl. Forssk. Fl. Aegypt. Arab. : 196 (1994).

Type: Ethiopia, Prope Aguar in Prov. Modat; Schimper

Sida cordifolia Forssk., Fl. Aegypt.-Arab.:124; CXVI no. 410 (1775), non L. (1753).

Abutilon cornutum Dalzell ex T. Cooke, Fl. Bombay 1 : 98 (1901).

\section{Distribution:}

Rare in wadi beds and sandy plains of Gebel Elba district, Galala Desert and Dakhla Oasis. Known from Trop. Africa, Arabian Peninsula to Iran, Pakistan and Australia. 


\section{Selected specimens:}

(Dg) Wadi Angabiya, Suez Road, 28.1.1956; Imam s.n. (CAI) - (O) Dakhla Oasis, Ain Fatima, 9.6.1961; V.Täckholm et al. s.n. (CAI) - (Sa) West slopes of Gebel Asotriba, 28.1.1962; V.Täckholm et al. s.n. (CAI).

3.3. Abutilon fruticosum Guill. \& Perr. in Guill. et al., Seneg. Tent. 1 : 73 (1831); Asch. \& Schweinf., Ill. Fl. Égypte : 52 no. 208 (1887); Ramis, Best. - Tabell. Fl. Aegypt. : 134 (1929); Täckh., Stud. Fl. Egypt ed. 1 : 230 (1956); et ed. 2 : 354 (1974).

Type: Described from senegal, 1821; Royer $(\mathrm{K})$.

Sida denticulata Fresen., Beitr. Fl. Aegypt. 2 : 182 (1834).

Abutilon microphyllum A. Rich., Tent. Fl. Abyss. 1 : 70 (1847).

A. denticulatum (Fresen.) Webb., Fragm. Fl. Aethiop. Aegypt. : 51 (1854); Asch. \& Schweinf., Ill. Fl. Égypte, 52 no. 208 (1887); Muschl., Man. Fl. Egypt 1 : 632 (1912).

\section{Distribution:}

Fairly common in sandy plains and wadi beds of Gebel Elba district, rare in mountainous Sinai and Red Sea Coasts. Known from Trop. Africa, Arabian Peninsula, Pakistan and Iran.

\section{Selected specimens:}

(S) Sinai, Wadi Isla, 4.2.1940; Hassib s.n. (CAI) - (R) Wadi El Faraied, Red Sea Coast, 7.2.1961; V.Täckholm et al. s.n. (CAI) - (Sa) Khor Yahameib, 22.1.1962; V.Täckholm et al. s.n. (CAI).

3.4. Abutilon pannosum (G. Forst.) Schlecht. in Bot. Zeit. 9 : 828 (1851); Täckh., Stud. Fl. Egypt ed. 1 : 226 (1956); et ed. 2 : 354 (1974); Hepper \& Friis, Pl. Forssk. Fl. Aegypt. - Arab. : 196 (1994).

Basionym : Sida pannosa G. Forst., in Comment. Soc. Reg. Sci. Goett. ser. 2, 9 : 62 (1789).

Type: Not known.

Sida glauca Cav., Ic. Descr., Pl. Hisp. 1 : 8, t. 11 (1791).

Sida mutica Delile, Descr. Égypte, Hist. Nat. : 68 no. 633 (1813), nom. nud. ex DC., Prodr. 1 : 470 (1824); Delile, Cent. Itin Caill. 60 no. 45 (1826).

Abutilon glaucum (Cav.) Sweet, Hort. Brit. : 54 (1826).

Abutilon muticum (Delile ex Dc.) Sweet, Hort. Brit. ed. 2 : 65 (1830); Boiss., Fl. Orient. 1:936 (1867); Asch. \& Schweinf., Ill. Fl. Égypte : 52 no. 211 (1887); Muschl., Man. Fl. Egypt 1 : 633 (1912). 


\section{Distribution:}

Weed in cultivations of the Nubian sector of the Nile land, its desert outskirts, the Oases and Gebel Elba district. Known from Trop. Africa to India and Afghanistan, eastwards to the Mediterranean.

\section{Selected specimens:}

(Nv) Qena, E Nag El Bayada, 9.1.1931; Basta s.n. (CAIM) - (Nn) Kom Ombo desert, 20.1.1927; G.Täckholm s.n. (CAI) - (O) El Kharga, Doush village, 24.11.1983; Barakat s.n. (CAI) - (R) Foot of Gebel Samiuki, Red Sea Coast, 6.2.1961; V.Täckholm et al. s.n. (CAI) - (Sa) Gebel Elba, Wadi Shallal, 24.1.1962; V.Täckholm et al. s.n. (CAI).

The species was reported by Danin et al. (1985: 290 \& $1993: 171)$ from southern Sinai [40 km N. of Sharm El Sheikh, 24.1.1969; Tadmor s.n.; Wadi Watir, $5 \mathrm{~km}$ SE of Ain Furtage, 27.3.1968; Danin, Tadmor \& Orshan s.n.].

3.5. Abutilon figarianum Webb., Fragm. Fl. Aethiop. Aegypt. : 52 (1854); Täckh., Stud. Fl. Egypt ed. 1 : 230 (1956); et ed. 2 : 354 (1974).

Type: Nubia in Cordofano ad montem Arasch, 14.10.1839; Kotschy. 180 (K).

\section{Distribution:}

Recorded once from the sandy coastal plains of the Red Sea Coast. Known from Arabian Peninsula, the Sudan and Ethiopia.

\section{Selected specimens:}

(R) Wadi Gweibba, Red Sea Coast, 9.6.1960; V.Täckholm s.n. (CAI).

4. Sida L., Sp. P1. ed. 1 : 683 (1753).

A large genus of about 200 species, widely distributed throughout the warmer regions of the world especially N America.

Note: Täckholm (1974:353) reported the occurrence of Sida ovata Forssk. and S. rhombifolia L. as very rare species in Gebel Elba district, and mountainous Sinai respectively. No. specimens were seen by the writers who believe that the occurrence of these species in Egypt is doubtful.

\section{Key to the species}

1. a. Leaves hirsute - villous beneath, flower up to $6 \mathrm{~mm}$ diam, petiole 2-12 $\mathrm{mm}$ long; awn of mericarps $2 \mathrm{~mm}$ long

b. Leaves glabrous - pilose beneath, flower up to $15 \mathrm{~mm}$ diam, petiole $10-30 \mathrm{~mm}$ long; awn of mericarps 4-6 mm long ...... 
4.1. Sida alba L., Sp. Pl. ed. 2 : 960 (1763); Täckh., Stud. Fl. Egypt ed. 1 : 229 (1956) et ed. 2 : 353 (1974); Hepper \& Friis, Pl. Forssk. Fl. Aegypt. Arab. : 199 (1994).

Type: Described from India.

Stewartia corchoroides Forssk., Fl. Aegypt. Arab.:126, LXX no. 347, IV no. 98 (1775).

Sida spinosa sensu Vahl (1791) non L. (1753); Delile Descr. Egypt, Hist. Nat. 2 : 68 (1813); Asch. \& Schwenf., Ill. Fl. Égypte : 52 no. 207 (1887); Sickenb., Mém. Inst. Égypt. 4(2):191 (1901); Muschl., Man. Fl. Egypt 1 : 629 (1912); Ramis, Best. Tabell. Fl. Aegypt.: 134 (1929).

\section{Distribution:}

Common summer weed in the farmlands of Egypt. Known from tropical and subtropical regions of the world.

\section{Selected specimens:}

(Nv) Fariskur, 28.8.1970; Imam et al. s.n. (CAI) - Lahoun, El Fayium, 15.9.1991; S.Araffa s.n. (CAI) - (Nn) Kom Ombo, 2.7.1967; El Hadidi s.n. (CAI) - (O) Bahariya Oasis, 11.9.1971; Imam s.n. (CAI) - Dakhla Oasis, 4.3.1934; Shabetai 4362 (CAIM).

4.2. Sida acuta Burm. fil., Fl. Ind. : 147 (1768); Täckh., Stud. Fl. Egypt ed. 2 : 353 (1974).

Type: Java, s. coll., s.n. (G) (Selected by Waalkes $1966: 187-8$ ).

\section{Distribution:}

Cultivated and escaped. Known from the Tropics.

\section{Selected specimens:}

(Nv) : Cairo, Manial Palace Garden, 7.4.1968; El Mahdi 12 (CAI).

* 5. Malvella Jaub. \& Spach, Illustr. Pl. Orient. 5 : 47 tab. 444 (1855).

Sida L. Sect. Pseudomalvastrum A.Gray, Mem. Amer. Acad. Arts n.s. 4 Pl. Frendl.) : 23 (1849).

A monotypic genus of only one species (M. sherardiana) known from Mediterranean to Caucasia.

Note: Malvella sherardiana is a new record to the flora of Egypt from Dakhla Oasis. It was erroneously identified as Malva rotundifolia $\mathrm{L}$. which is a different taxon. 
M. N. El-Hadidi, H. A. Hosni, A.H. El-Hadidy \& S. Araffa

* 5.1. Malvella sherardiana (L.) Jaub. \& Spach, Ill. Pl. Orient. 5:46 (tab. 444(1855).

Basionym : Malva sherardiana L., Sp. Pl. ed. 2 : 1675 (1763).

Sida sherardiana (L.) Benth., Four. Linn. Soc. Bot. 6 : 101 (1862).

\section{Distribution:}

Waste places and fields; recorded once from Dakhla Oasis of Egypt. Known from the Mediterranean to S.W. Asia.

\section{Selected specimens:}

(O) Dakhla Oasis, 6.3.1934; Shabetai Z4363 (CAIM).

6. Malvastrum A.Gray, Mem. Amer Acad. Arts. ser. 2, $4: 21$ (1849) - nom. conserv.

A genus of 12 species widely distributed throughout tropics and subtropics especially America.

6.1 Malvastrum coromandelianum (L.) Garcke in Bonplandia 5 : 295 (1857); Täckh., Stud. Fl. Egypt ed. $1: 226$ (1956) et ed. $2: 351$ (1974).

Basionym : Malva coromandelianum L., Sp. Pl. ed. 1 : 687 (1753).

Type: Specimen no. 870/3 (LINN) [Lectotype selected by Waalkes: 152 (1966)].

Malvastrum tricuspidatum (R. Br. ex W. T. Aiton) A. Gray, Plantae Wrightianae 1 : 16 (1852).

Malva tricuspidata R. Br. ex W. T. Aiton, Hort. Kew. (2) 4 : 210 (1812).

\section{Distribution:}

Introduced and naturalized in the gardens around Cairo. Known from S America, Australia \& S Africa; elsewhere introduced and naturalized.

\section{Selected specimens:}

(Nv) Giza, Zoharia garden at El Gezira, 15.5.1927; G.Täckholm s.n. (CAI).

7. Malva L., Sp. Pl. ed. $1: 687$ (1753).

Alcea Mill., Gard. Dict. Abr. 4 : 45 (1754) non L. (1753).

A genus of about 100 species in Temperate regions of $\mathrm{N}$ hemisphere and $\mathrm{S}$ America, introduced into Australia. 


\section{Key to the species}

1. a. Leaves palmatisect \pm glabrous with entire margin; epicalyx of 2 bracteoles

b. Leaves orbicular - reniform or palmatipartite, sparsely or densely hairy, with dentate - serrate margin; epicalyx of 3 bracteoles

2. a. Flowers 5-7 mm diam.; petals $10-40 \mathrm{~mm}$ long, 4 times as long as sepals. Fruit $7-$ $10 \mathrm{~mm}$ diam

b. Flowers up to $5 \mathrm{~mm}$ diam.; petals $5-12 \mathrm{~mm}$, as long as or twice as long as sepals. Fruit up to $7 \mathrm{~mm}$ diam

3. a. Bracteoles linear; calyx lobes broadly ovate - roundish, apiculate; petals up to 6 $\mathrm{mm}$ long, as long as sepals; mericarps of elevated margins

b. Bracteoles elliptic-ovate or oblong; calyx lobes ovate-triangular, with acuteacuminate apex; petals 6-12 mm long, exceeding sepals in length; mericarps without elevated margins

4. a. Petals pale mauve, up to $8 \mathrm{~mm}$ long; mericarps smooth from the dorsal surface

b. Petals purple - violet, $10-12 \mathrm{~mm}$ long; mericarps deeply reticulate from the dorsal surface

3. M. nicaeensis

7.1. Malva aegyptia L., Sp. Pl. ed. 1 : 690 (1753); Delile, Descr. Égypte, Hist. Nat. 2 : 68 (1813); Asch. \& Schweinf., Ill. Fl. Égypte : 50 no. 818 (1887); Sickenb., Mém. Inst. Égypt. 4(2) : 191 (1901); Muschl., Man. Fl. Egypt 1 : 625 (1912); Ramis, Best. Tabell. Fl. Aegypt. : 133 (1929); Täckh., Stud. Fl. Egypt ed. 1 : 225 (1956); et ed. 2 : 349 (1974).

Type: Described from Egypt.

Malva aegyptia L. var. leicocarpa Iljin in Not. Syst. Herb. Hot. Petrop. 4: 26 (1923). M. leicocarpa (Iljin) Iljin, Fl. USSR 15 : 35 (1949).

M. iljinii Riedl. In Rech. f., Fl. Iran. 120 : 16 (1976).

\section{Distribution:}

Common on rocky slopes and sandy soils along the Mediterranean coastal land of Egypt. Known from N Africa, S Europe with extensions to S. W. Asia and Iran.

\section{Selected specimens:}

(M) Mersa Matrouh, 22.3.1975; V.Täckholm et al. s.n. (CAI) - Burg El Arab, Maruit, 14.3.1951; A.Amin s.n. (CAI) - Al Abtal station, N El Arish, 18.3.1955; El Hadidi s.n. (CAI) - (Dg) Suez road, 21.2.1960; Khattab s.n. (CAIM). 
7.2. Malva parviflora L., Demonstr. : 18 (1753) et Ameon. Acad. 3 : 413 (1756) et Sp. Pl. ed. 2 : 969 (1763); Delile, Descr. Égypte, Hist. Nat. 2 : 68 (1813); Asch. \& Schweinf., Ill. Fl. Égypte : 51 no. 820 (1887); Sickenb., Mém. Inst. Égypt. 4(2) : 191 (1901); Muschl., Man. Fl. Egypt 1 : 626 (1912); Ramis, Best. - Tabell. Fl. Aegypt. : 133 (1929); Täckh., Stud. Fl. Egypt ed. 1 : 225 (1956) et ed. 2 : 349 (1974); Hepper \& Friis, Pl. Forssk. Fl. Aegypt. Arab. : 198 (1994).

Type: Described from Barbary, specimen no 370/17 (LINN).

Malva microcarpa Desf. ex Pers., Syn. Pl. 2 : 251 (1806)

M. mareotica Delile ex Dc. Prodr. 1 : 433 (1824)

M. parviflora var. cristata Boiss., Fl. Orient. 1 : 821 (1867)

M. parviflora var. microcarpa (Desf. ex Pers.) Fiori Paol, Fl. Anald. Ital. 2 : 268 (1900-02).

M. rotundifolia sensu Forssk. (1775) non L. (1753).

\section{Distribution:}

A common weed of cultivation, desert plains and moist habitats all over the country. Known from the Mediterranean, North Africa, eastward to Iran and Afghanistan. Naturalized in N. America.

\section{Selected specimens:}

(M) Sallum, 24.5.1963; V.Täckholm s.n. (CAI) - Amriya W. of Alexandria, 26.3.1954, L.Boulos s.n. (CAI) - ElArish, 20.3.1928; G.Täckholm s.n. (CAI) - (DI) Wadi EL Natroun, 18.7.1968; El Hadidi et al. s.n. (CAI) - (Di) along the road to Ismailia, 18.71968; El Hadidi et al. s.n. (CAI) - (Dg) Wadi Angabiya, Suez road, 30.1.1953; V.Täckholm s.n. (CAI) - (Nv) Kafr El Ezz, near Mansoura, 8.4.1977; Chertek et al. s.n. (CAI) - (Nn) Kom Ombo, 21.1.1927; G.Täckholm s.n. (CAI) - (O) Bahariya Oasis, 28.2.1978; Abdel Ghani 252 (CAI) - (S) Wadi Feiran, Sinai, 3.5.1930; Drar s.n. (CAI) N. Abu Zeneima, 13.4.1962; Abdallah 534 (CAIM) - (Sa) Wadi Oolak, 27.1.1962; V.Täckholm et al. s.n. (CAI).

7.3. Malva nicaeensis All., Fl. Pedem. 2 : 40 (1785); Asch. \& Schweinf., Ill. Fl. Égypte, Suppl. : 751 (1889); Sickenb., Mém. Inst. Égypt. 4 (2) : 191 (1901); Muschl., Man. Fl. Egypt 1 : 626 (1912); Ramis, Best. Tabell. Fl. Aegypt. : 133 (1929); Täckh., Stud. Fl. Egypt ed. 1 : 226 (1956) et ed. 2 : 349 (1974).

Type: France, Prope Portum di Limpia, Bellardi.

\section{Distribution:}

Rare weed in waste places and roadsides along the Mediterranean coastal land of Egypt. Known from the Mediterranean, N Africa, eastwards to Iran and Afghanistan. 


\section{Selected specimens:}

(M) Burg El Arab, 1.4.1952; Bot. Dept. Excursion s.n. (CAI).

7.4. Malva neglecta Wallr, Syll. Pl. Nov. 1 : 140 (1824).

Type: Not known.

Malva rotundifolia sensu Boiss., Fl. Orient. 1 : 820 (1867); Täckh., stud. Fl. Egypt ed. $1: 226$ (1956) et ed. $2: 349$ (1974)

\section{Distribution:}

Wadi beds, waste places and rocky ground of mountainous Sinai. Known from Palestine to Iran and Afghanistan, introduced to United States of America.

\section{Selected specimens:}

(S) Wadi Feiran, 24.4.1961; V.Täckholm et al. s.n. (CAI) - Gebel Katherine, S Sinai, 7.5.1939; Drar 342 (CAIM).

7.5. Malva sylvestris L., Sp. Pl. ed. 1 : 689 (1753); Forssk., Fl. Aegypt. Arab. VIII no. 164, XIV no. 54 (1775); Asch. A Schweinf., Ill Fl. Égypte : 15 no. 819 (1887); Sickenb., Mem. Inst. Egypt 4(2) : 191 (1901); Muschl., Man. Fl. Egypt 1: 626 (1912); Ramis, Best. - Tabell. Fl. Aegypt. : 133 (1929); Täckh., Stud. Fl. Egypt ed. 1:226 (1956) et ed. 2:349 (1974).

Type: Described from Europe; Specimen no. 870/22 (LINN).

Malva ambigua Guss., Fl. Sicul. Prodr. 2 : 331 (1828).

M. sylvestris var. mauritana (L.) Boiss., Fl. Orient. 1 : 819 (1867).

M. erecta C. Presl, Fl. Sicula : 75 (1826).

M. sylvestris var. oxyloba Post, Jour. Linn. Soc. Lund 24 : 224 (1888) et Fl. Pal. ed. $1: 178$ (1896).

\section{Distribution:}

Common weed of the coastal plains along the Mediterranean, wadi beds of Libyan and Isthmic Deserts of Egypt. Known from the Mediterranean, S Europe and N Africa to SW Asia and Iran; introduced in the United States of America.

\section{Selected specimens:}

(M) Sallum, Wadi El Ramlah, 14.4.1934; Shabetai 3071 (CAIM) - (Dl) Cairo Alexandria road, S of El Amriya, 12.3.1991; S.Araffa a.n. (CAI) - (Di) Ain El Gedirat, 6.4.1936; Drar s.n. (CAIM). 
8. Althaea L., Sp. Pl. ed. 1 : 686 (1753)

A genus of about 12 species distributed in W.Europe, NE Siberia, C. Asia and the Mediterranean region.

8.1. Althaea ludwigii L., Mant. Pl. : 98 (1771); Delile, Descr. Egypte, Hist Nat. : 68 (1813); Asch. \& Schweinf., Ill. Fl. Egypte : 51 no. 204 (1887); Sickenb., Mém. Inst. Égypt. 4(2) : 191 (1901); Muschl., Man. Fl. Egypt 1 : 627 (1912); Ramis, Best. Tabell. Fl. Aegypt. : 133 (1929); Täckh., Stud. Fl. Egypt ed. 1 : 228 (1956) et ed. 2 : 353 (1974).

Type: Specimen no. 868/4 (LINN).

\section{Distribution:}

Very rare in wadi beds and moist places of western Mediterranean coastal land, the Eastern Desert and the Oases. Known from N. Africa, eastwards to Iran and India; also known from $\mathrm{S}$ Africa.

\section{Selected specimens:}

(M) Maruit, Dabaá, 3.5.1955; Bot. Dept. Excursion s.n. (CAI) - (Di) Wadi Hereidin, S El Arish, 5.4.1939; Drar s.n. (CAIM) - (O) Dakhla Oasis, 15.3.1929; Drar 62/901 (CAIM).

The species was reported by Danin et al. (1985 : 291) from mountainous Sinai; no specimens were available for this study.

9. Alcea L., Sp. Pl. ed. $1: 687$ (1753)

A genus of 60 species in Mediterranean to Central Asia.

Note: Alcea apterocarpa (Fenzl.) Boiss. is an E. Mediterranean element, known mainly from Turkey with slight extension southward to Palestine and Syria. The species was reported by Täckholm (op.cit.) from Sinai. It was not reported by Abdallah et al. (1984) or Danin et al. (1985) and its occurrence in Sinai is doubtful.

Alcea rosea $\mathrm{L} .=$ Althaea rosea $(\mathrm{L}$.$) Cav.) is a cultivated ornamental in gardens which was$ reported by Täckholm (op. cit. 353 ) as an escape from cultivation in Sinai.

\section{Key to the species}

1. a. Stem well developed, with remote flowers

1. A. striata

b. Stemless or short stemmed with crowded flowers

2. A. acaulis

9.1. Alcea striata (DC.) Alef., Österr. Bot. Zeit. 12 : 253 (1862); subsp. striata. Sickenb., Mém. Inst. Égypt. 4(2) : 191 (1901); Täckh., Stud. Fl. Egypt ed. 2 : 351 (1974).

Basionym: Althaea striata DC., Prodr. 1 : 437 (1824); Muschl., Man. Fl. Egypt 1 : 628 (1912); Täckh., Stud. Fl. Egypt ed. 1 : 228 (1956).

Type: Described from a specimen cultivated in the Hortus Celsius.

Althaea hausskenechtii Boiss., Fl. Orient. 1 : 830 (1867). 
Note: Täckholm (1974:351) as well as El Hadidi \& Fayed (1994/95 : 96) and Boulos (1995 : 91) reported Alcea striata as a multiform species represented by subsp. striata and subsp. rufescens (Boiss.) Cullen. Careful examination of the available specimens proved that only the typical subspecies viz. A.striata subsp. striata is represented in Egypt.

\section{Distribution:}

Rare in wadis of mountainous Sinai. Known from Palestine, syria, Turkey and Arabian Peninsula.

\section{Selected specimen:}

(S) Deir El Arbain, Sinai, 12.5.1956; El Hadidi s.n. (CAI).

9.2. Alcea acaulis (Cav.) Alef., Österr. Bot. Zeit. 12 : 251 (1862); Asch. \& Schweinf., Ill. Fl. Égypte : 52 no. 205 (1887); Muschl., Man. Fl. Egypt 1 : 628 (1912); Täckh., Stud. Fl. Egypt ed. 2 : 351 (1974).

Basionym: Althaea acaulis Cav., Diss. 2 : 93 t. 27 (1786); Täckh., Stud. Fl. Egypt ed. $1: 228$ (1956).

Type: Not known

Note: Alcea acaulis is an E. Mediterranean element, which was reported by Täckholm (1974:351) as a rare species from W. Mediterranean coast of Egypt. The species was also recorded earlier by Ascherson \& Schweinfurth (1887:52) and Muscher (1912:628) without locality and by Dinsmore (1932:240) from Ain Musa (Sinai) and by Danin (1973 : 26) from Gebel El Raha (Sinai). Although no specimens were available for this revision, the species geographical range favour its occurrence in Egypt.

\section{Distribution:}

Known from Syria, Lebanan, Palestine and Turkey.

10. Lavatera L., Sp. Pl. ed. 1 : 690 (1753)

Anthema Medik, Malv. : 41 (1787)

Stegia DC. In Lam. \& DC., Fl. Franc. (ed.3) 4 : 835 (1805).

A genus of about 45 species in temperate and subtropical regions extending to C. Asia and Australia.

10.1. Lavatera cretica L., Sp. Pl. ed. 1 : 691 (1753); Delile, Descr. Égypt. Hist Nat. 2 : 68 (1813); Asch. \& Schweinf., Ill. Fl. Égypt. : 52 no. 213 (1887); Sickenb., Mém. Inst. Égypte 4(2) : 191 (1901); Muschl., Man. Fl. Egypt 1 : 629 (1912); Ramis, Best.-Tabell. Fl. Aegypt. : 133 (1929); Täckh., Stud. Fl. Egypt ed. 1 : 226 (1956) et ed. 2 : 351 (1974).

Type: Described from Crete; specimen no. 871/10 (LINN). 


\section{Distribution:}

Rare weed in waste ground and cultivations of the Mediterranean coastal land as well as the Nile Delta. Known from the Mediterranean, N. Africa eastward to Arabia.

\section{Selected specimens:}

(M) Burg El Arab, 22.3.1956; El Hadidi s.n. (CAI) - (Nv) Giza, 22.5.1964; Shabetai 2908 (CAIM).

11. Pavonia Cav., Diss. 2 : App. 2 (1786), nom. conserv.

Lass Adans., Fam. Pl. 2 : 400 (1763)

A large genus of about 200 species distributed in Tropical and subtropical regions of both hemispheres.

Note: Täckholm (1974:355), El Hadidi \& Fayed (1994/95 : 98) and Boulos (1995 : 93) reported three species of Pavonia from Egypt without certainty, based on Ulbrich's (1920) monograph viz. P.arabica Steud. \& Hochst ex Boiss., P.hirsuta Guill. \& Perr. and P.burchelli (DC.)R.A.Dyer. These species are of limited geographical distribution (Arabian Peninsula and Abyssinia) and their occurrence in Egypt is doubtful.

\section{Key to the species}

1. a. Fruit glabrous, mericarps broadly winged 1. P. kotschyi

b Fruit pubescent, mericarps wingless or narrowly winged 2. P. triloba.

11.1. Pavonia kotschyi Hochst ex Webb., Frag. Fl. Aethiop. 34 (1854); Täckh., Stud. Fl. Egypt ed. 1:230 (1956) et ed. 2:355 (1974).

Type: Ad Pagum Cordofonum, Abu Gerad, 18.9.1939; Kotschy 12 (K).

\section{Distribution:}

Rare in wadi beds and rocky ground of Gebel Elba district. Known from Arabian Peninsula and E. Africa.

\section{Selected specimens:}

(Sa) Wadi Kansisrob, 25.1.1929; Shabetai 2677 (CAIM).

11.2. Pavonia triloba Guill. \& Perr., Fl. Senegamb. Tent. 1 : 50 (1831); Täckh., Stud. Fl. Egypt ed. 1 : 231 (1956); et ed. 2 : 355 (1974).

Type: Crescit in Collibus Sabulosis siccis prope Dagama provincia, Walo. 


\section{Distribution:}

Rare in wadi beds and mountain slopes of Gebel Elba district. Known from Sudan and Arabian Peninula.

\section{Selected specimens:}

(Sa) Gebel Elba, 23 - 27.1.1929; G.Täckholm s.n. (CAI) - Gebel Karam Elba mountain, 7.2.1962; V.Täckholm s.n. (CAI).

\section{References:}

Abdallah, M., Saad, F., Eweida, A. \& Mahmoud, M. (1984). Materials from CAIM Herbarium II. Flora of the Sinai Peninsula. Notes A.R.C. Herb. Egypt 6: 156 158.

Ascherson, P. \& Schweinfurth, G. 1887. Illustration de la florer d'Égypte. Mém Inst. Égypte 2(1) : 51 - 53.

Bentham, G. \& Hooker, J. D. 1862. Genera Plantarum 1 : 195 - 204. London.

Boulos, L. 1995. Flora of Egypt, Checklist : 91 - 93 . Al Hadara Publishing, Cairo.

Bremer, K., Bremer, B. \& Thulin, M. 1998. Introduction to phylogeny and systematics of flowering plants: 66-68. Department of systematic Botany, Uppsala University.

Danin, A. M. 1973. Contribution to the Flora of Sinai II. Isr. Jour. Bot. 22 : 26.

1993. Contributions to the Flora of Sinai IV. Comment on the "Annotated list of the Flora of Sinai" (1989). Willdenowia 23 : 167 - 175.

- Schmida, A. \& Liston, A. 1985. Contributions to the Flora of Sinai III. Checklist of the species collected and recorded by the Jersualem team 1967 1982 . Willdenowia 15(1) : $290-291$

Dinsmore, J. E. in Post, G. \& Dinsmore, J. E. 1932. Flora of Syria, Palestine and Sinai : 240. Beirut.

El Hadidi, M. N. 1980. An outline of the planned flora of Egypt, in M.N. El Hadidi (ed.) Flora of Egypt. Taeckholmia add. ser. 1:9-10.

\& Fayed, A. A. 1994/95. Materials for Excursion Flora of Egypt. Taeckholmia $15: 96-98$.

Hutchinson, J. B. 1967. Genera of Flowering plants 2 : 536 - 567. London.

1969. Evolution and phylogeny of Flowering plants : 253 - 257. London.

Muschler, R. 1912. Manual Flora of Egypt 1: 624 - 639. Berlin.

Schumann, K. 1895. Malvaceae in A. Engler \& K. Prant 1. Natürlichen Pflanzenfamilien 3(6) : 30 - 35. Leipzig - Cobenhagen. 
Täckholm, V. 1956. Students' Flora of Egypt ed. 1:225 - 232. Anglo-Egyptian Bookshop, Cairo.

1974. Students' Folra of Egypt ed. 2:348 - 356. Cairo University, Cairo.

Ulbrich, E. 1920. Monographie der afrikanischen Pavonia Arten nebst Uebersicht über die gane Gattung. Bot. Jahrb. 57:54-184.

Zohary, M. 1963a. Taxonomical studies in Alcea of South Western Asia, Part 1. Bull. Res. Counc. of Israel 11(4) : 210 - 229.

1963b. Taxonomical studies in Alcea of South Western Asia, Part 2. Isr. J. Bot. 12(1) : 1 - 26. 